THe ASTrophysical Journal SUPPLEMENT SERIES, 90:577-581, 1994 February

(C) 1994. The American Astronomical Society. All rights reserved. Printed in U.S.A.

\title{
ELECTRON ACCELERATION AT QUASI-PARALLEL SHOCKS IN THE SOLAR CORONA AND ITS SIGNATURE IN SOLAR TYPE II RADIO BURSTS
}

\author{
G. MANN \\ Astrophysikalisches Institut Potsdam, 14552 Tremsdorf, Germany \\ AND \\ H. LÜHR \\ Institut für Geophysik und Meteorologie der Technischen Universität, 38106 Braunschweig, Germany \\ Received 1993 February 17; accepted 1993 June 8
}

\begin{abstract}
Recently, strong large amplitude magnetic field structures (SLAMS) have been observed as a common phenomenon in the vicinity of the quasi-parallel region of Earth's bow shock. A quasi-parallel shock transition can be considered as a patchwork of SLAMS. Using the data of the AMPTE/IRM magnetometer the properties of SLAMS are studied. Within SLAMS the magnetic field is strongly deformed and, thus, the magnetic field geometry is locally swung into a quasi-perpendicular regime. Therefore, electrons can locally be accelerated to high energies within SLAMS. Assuming that SLAMS also exist in the vicinity of supercritical, quasi-parallel shocks in the solar corona, they are able to generate radio radiation via the enhanced Langmuir turbulence excited by the accelerated electrons. Since SLAMS are connected with strong density enhancements, the aforementioned mechanism can explain the multiple-lane structure often occurred in solar Type II radio bursts.
\end{abstract}

Subject headings: acceleration of particles - Earth — shock waves - Sun: corona - Sun: radio radiation

\section{INTRODUCTION}

Collisionless shocks play an important role in astrophysics because they are reasponsible for accelerating particles to high energies (Fermi 1949; Axford 1981). Earth's bow shock is the collisionless shock wave in space by most observed extraterrestial in situ measurements (cf. as a review Kennel et al. 1985). Because of its curvature it has a quasi-perpendicular and quasiparallel region. In both cases the shock is accompanied by a great variety of high energetic particle populations and upstream wave phenomena (cf. as a brief review Tsurutani \& Rodriguez 1981). In contradiction to Earth's bow shock and interplanetary shocks (Kennel et al. 1982) shock waves in the solar corona can be observed only by remote sensing techniques, e.g., radioastronomical methods, where they manifest in solar Type II radio bursts. Coronal shock waves can be originated by solar flares or coronal mass ejections.

Solar type II radio bursts appear as enhanced emission stripes, also called "backbone" with a slow drift from high to low frequencies of $\leq 1 \mathrm{MHz} \mathrm{s}^{-1}$ in dynamic radio spectra (Krüger 1979). The emission is often repeated at the second harmonic. Type II bursts show a great variety of fine structures, e.g., band splitting, multiple-lane structures and herringbones (cf. McLean 1985). Some Type II bursts show emission features rapidly drifting to low and high frequencies from the main emission stripe (backbone) and resemble Type III bursts. These rapidly drifting emission stripes are usually called "herringbones." They are interpreted as electrons accelerated at the shock front and propagating in the up- and downstream regions, i.e., in regions of decreasing and increasing density, respectively. Sometimes the backbone is doubled in emission stripes separated by a few megaHertz in both the fundamental and harmonic band. This is called "band splitting." Furthermore, the backbone is sometimes split into two or more emission bands with slightly different drift rates, i.e., the backbone show a "multiple-lane structure."

It is generally assumed that the radio radiation is mediately generated by suprathermal electrons. These electrons can excite high-frequency electrostatic waves, e.g., Langmuir waves, which scatter on ion density fluctuations and/or low-frequency ion waves in order to convert into escaping electromagnetic waves with frequencies slightly above the local electron plasma frequency. Furthermore, Langmuir waves can also interact with themselves, coalescing into electromagnetic waves with frequencies near the double of the electron plasma frequency. Thus, the former and latter mechanisms are responsible for the fundamental and harmonic radiation (cf., e.g., Krüger 1979), respectively.

Recently, Benz \& Thejappa (1988), with the aim of a better physical understanding of solar Type II radio emission, applied the new observational results of Earth's bow shock, in particular those resulting from the ISEE mission, to coronal shock waves. Basically, they assumed that despite the different parameters of Earth's bow shock and coronal shocks the basic physics of collisionless shocks is essentially the same. They argued that the exciter of the backbone is different from the herringbone source. The backbone is thought to be generated by the coalescence of upper hybrid waves with low-frequency ion waves. Both waves are excited by high energetic electrons and ions, respectively. Suprathermal electrons and high energetic ions are observed upstream of Earth's bow shock (cf. Parks et al. 1981). Both are accompanied by high-frequency plasma oscillations (e.g., Langmuir waves) and ultra-low-frequency magnetohydrodynamic waves, where the high-fre- 
quency plasma oscillations are usually most intense near the upstream boundary of the electron foreshock (cf. Gurnett 1985 as a review).

Holman and Pesses (1983) have suggested that the shock drift acceleration mechanism is responsible for the high energetic electrons, which are needed for producing a Type II burst. They have argued that the production of Type II emission via shock drift acceleration of electrons requires an angle between the shock normal and the upstream magnetic field greater than $80^{\circ}$, i.e., a quasi-perpendicular shock region. Furthermore, they have interpreted the appearance of herringbone structures with and without the backbone, the appearance of the backbone without herringbones and the band splitting by means of a curved shock front propagating through the corona. Consequently, the shock front takes different angles to the upstream magnetic field and the coronal density gradient resulting in the different Type II burst features aforementioned.

Recently, short large amplitude magnetic structures (SLAMS) have been observed in the vicinity of the quasi-parallel region of Earth's bow shock (Thomsen et al. 1990; Schwartz et al. 1992). Schwartz \& Burgess (1991) argued that a quasiparallel collisionless shock transition should be regarded as a patchwork of SLAMS. Using the data of the magnetometer aboard the $A M P T E / I R M$ satellite it was shown that the magnetic field within SLAMS is locally swung into a quasi-perpendicular geometry (Mann \& Lühr 1992). Thus, electrons can be accelerated to high energies within SLAMS via the shock drift acceleration mechanism. Assuming that SLAMS also appear in the vicinity of quasi-parallel shock waves in the solar corona, these SLAMS can accelerate electrons which are able to excite Langmuir waves converting into escaping electromagnetic waves.

In $\S 2$ we intend to present the properties of SLAMS. The results of shock drift acceleration are briefly reviewed in $\$ 3$. In $\S 4$ we will discuss the possible acceleration of electrons at SLAMS occurring at coronal shock waves and their relevance to solar Type II radio bursts.

\section{PROPERTIES OF SLAMS}

During the time interval 10:20-11:05 UT on 1984 October 30 the $A M P T E / I R M$ satellite experienced several quasi-parallel, supercritical crossings of Earth's bow shock, where isolated and embedded SLAMS have been observed in the up- and downstream region (Schwartz et al. 1992), respectively. SLAMS are characterized as well-defined single magnetic structures with large amplitudes of $\sim 2$ or more times the background field and short durations of typically $10 \mathrm{~s}$. Here, we restrict ourselves to SLAMS appearing in the upstream region.

Figure 1 shows the magnetic field and particle density data of a typical isolated SLAMS occurring on 11:01:25 UT. The data were recorded by the magnetometer (Lühr et al. 1985) and the 3D plasma instrument (Paschmann et al. 1985) aboard the $A M P T E / I R M$ satellite. The magnetic field components are displayed in minimum variance coordinates which have been computed over the period 11:01:22-11:01:40 UT. The peak magnitude amounts to $B_{\max }=22 n T$, where the background field takes a value of $B_{0}=7.6 n T$, i.e., $\left(B_{\max }-B_{0}\right) / B_{0}=$ 1.9. The half-width defined at the $\left(B_{\max }+B_{0}\right) / 2$ level is $6.9 \mathrm{~s}$. As seen in the panel at the bottom of Figure 1, the SLAMS is accompanied by a density increase of $\left(N_{\max }-N_{0}\right) / N_{0}=2.0$

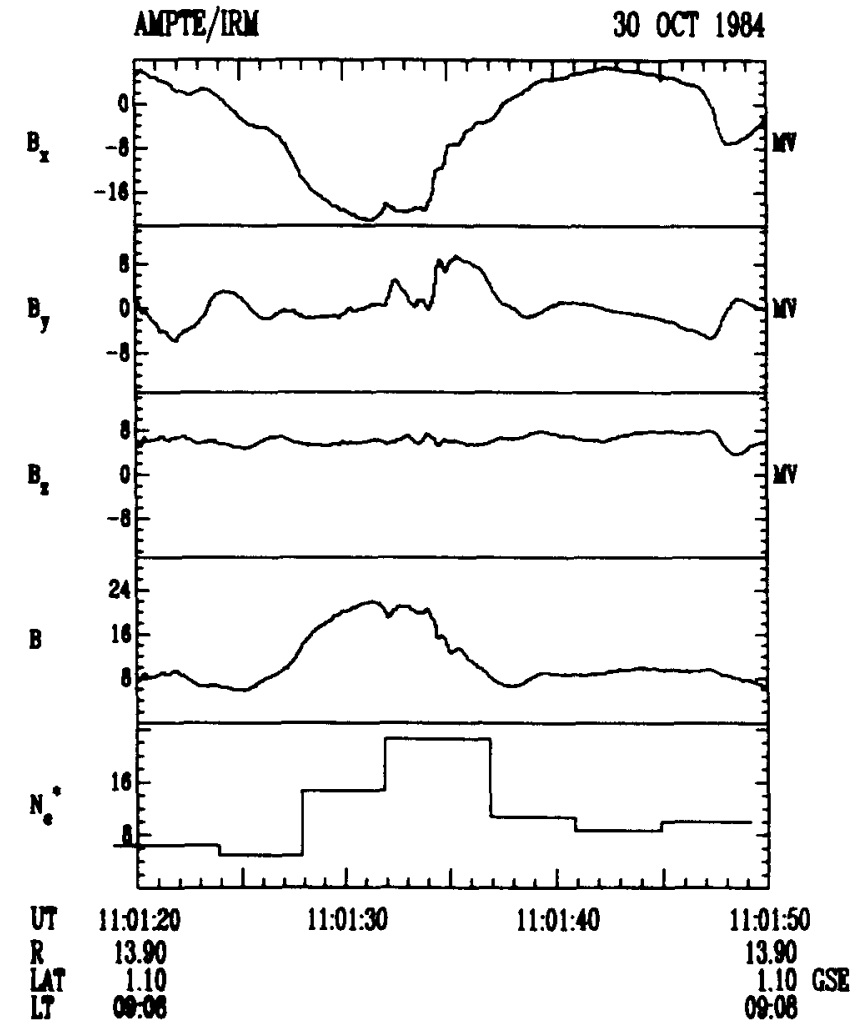

FIG. 1.-Magnetic field and particle density behavior of a typical isolated SLAMS occurring at 11:01:25 UT on 1984, October 30. The first four panels show the magnetic field components presented in a minimum variance frame and the field magnitude. Both are given in $n T$. The fifth panel represents the electron particle number density given in $\mathrm{cm}^{-3}$.

with $N_{\max }=22.6 \mathrm{~cm}^{-3}$ and $N_{0}=7.5 \mathrm{~cm}^{-3}$. This density enhancement represents an adiabatic compression of the background plasma. Employing the results of the minimum variance analysis we have found for the angles between the propagation direction and the background field, $\vartheta_{1, \boldsymbol{B}_{0}}$, and the mean internal field $\boldsymbol{B} \vartheta_{1, \boldsymbol{B}}$ as well as the angle between the shock normal $n_{s}$ and the mean internal field, $\vartheta_{n_{s}, B}, 35^{\circ}, 55^{\circ}$, and $55^{\circ}$, respectively.

Eighteen isolated SLAMS appearing in the upstream region have been statistically investigated with the aim of studying the local deformation of the magnetic field with respect to the shock normal, the propagation direction, and the background magnetic field. Thus, we find the following mean values and standard deviations:

1. Enhancement of the magnetic field magnitude:

$$
\left(\boldsymbol{B}_{\max }-\boldsymbol{B}_{0}\right) / \boldsymbol{B}_{0}=2.63 \pm 1.11 ;
$$

2. Enhancement of the particle number density:

$$
\left(N_{\max }-N_{0}\right) / N_{0}=1.3 \pm 0.8 ;
$$

3. Angle between the propagation direction of the SLAMS and the unperturbed magnetic field:

$$
\vartheta_{1, B_{0}}=33: 9 \pm 17^{\circ}: 2 \text {; }
$$


4. Angle between the propagation direction of the SLAMS and the mean internal magnetic field within the SLAMS

$$
\vartheta_{1,}=53: 4 \pm 13^{\circ} .4
$$

5. Angle between the shock normal and the mean internal magnetic field within the SLAMS

$$
\vartheta_{n_{s}, B}=52: 3 \pm 19: 7
$$

The distribution of the angles $\vartheta_{1, B}$ and $\vartheta_{n_{s}, B}$ are shown in Figures 2 and 3 . Note that the angle between the shock normal and the unperturbed upstream magnetic field lies in the interval $10^{\circ}-15^{\circ}$ (Schwartz et al. 1992).

The SLAMS are obliquely propagating along the undisturbed magnetic field. They can be considered as plane structures with a spatial width of typically $10^{8} \mathrm{~cm}$, i.e., $\sim 10$ ion inertial length, and a strong local deformation of the magnetic field (Mann \& Lühr 1992). This magnetic field deformation is accompanied by two antiparallel currents and electric fields directed across the propagation direction within the SLAMS. Thus, SLAMS represent local magnetic particle mirrors and are able to accelerate particles (cf. $\S 3$ ) in two different ways. On the one hand, the incident particles upstream of the SLAMS are accelerated at SLAMS and subsequently scattered back into the upstream region by a single encounter with the SLAMS. On the other hand, SLAMS can scatter particles, which are reflected from the shock front, back toward the shock front. Thus, these particles can gain energy by multiple encounters with the shock front and the upstream SLAMS (cf. Scholer 1985). Here, the former case will only be considered.

Within SLAMS the angle between the shock normal and the mean internal magnetic field is shifted into a quasi-perpendicular geometry. In 13 out of 18 cases ( $72 \%$ of all cases) this angle is greater than $45^{\circ}$ where the angle between the shock normal

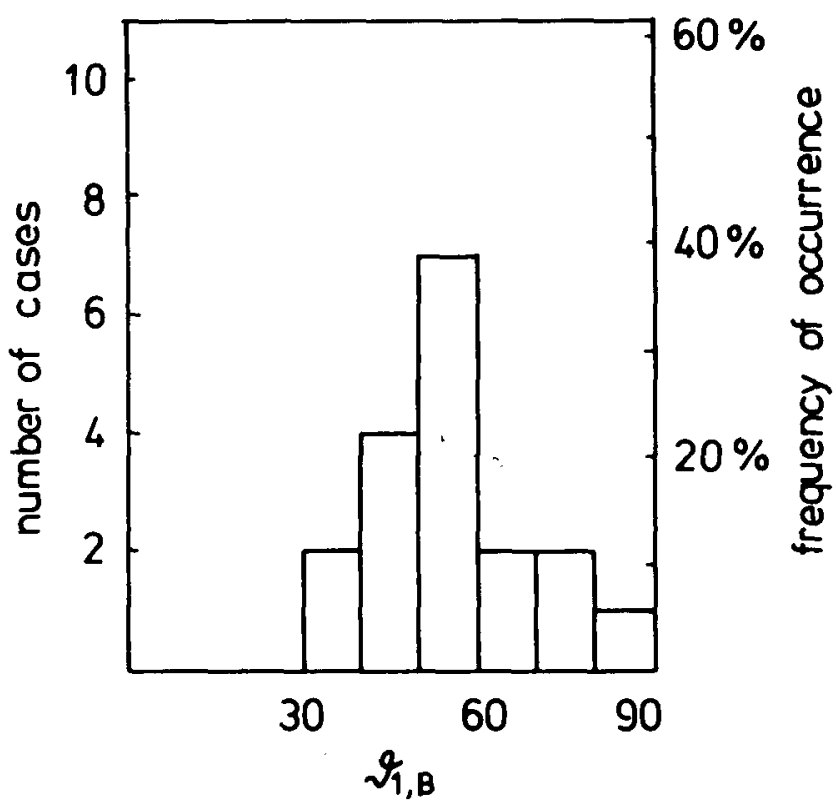

Fig. 2.-Histogram of the distribution of the angle $\vartheta_{1, B}$ between the propagation direction and the mean internal magnetic field of SLAMS studied.

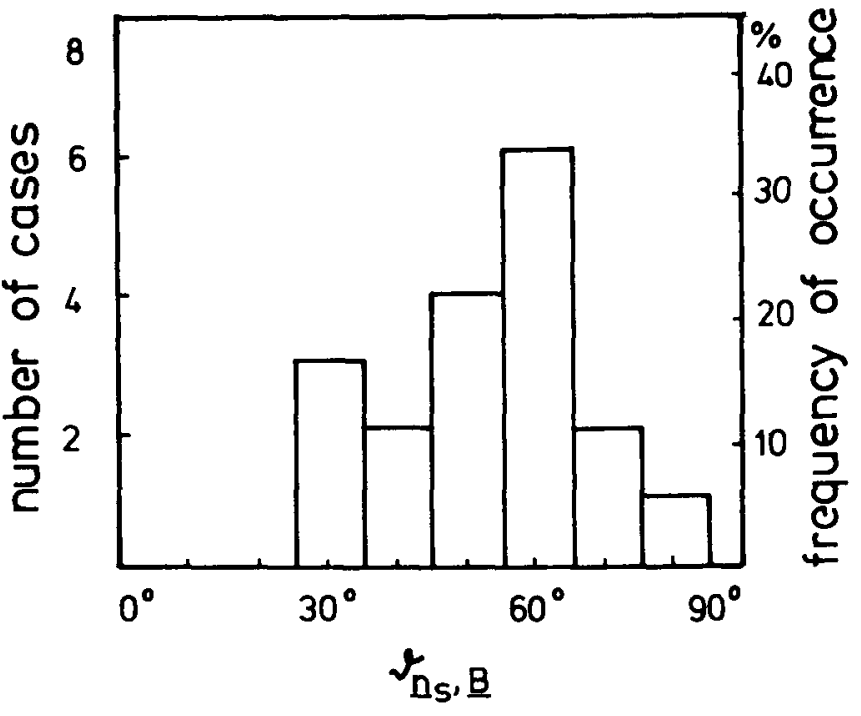

FIG. 3.-Histogram of the distribution of the angle $\vartheta_{n_{c} . B}$ between the shock normal and the mean internal magnetic field of the SLAMS investigated (cf. Mann \& Lühr 1992).

and the background field lies between $10^{\circ}$ and $15^{\circ}$ (Mann \& Lühr 1992).

Generally, SLAMS are growing from the ULF upstream waves which are propagating along the background magnetic field and are convected back to the bow shock by the solar wind (Schwartz et al. 1992). In the plasma rest frame their propagation speeds are well above the first magnetosonic velocity but lower than the shock speed (Schwartz et al. 1992). During their approach to the shock front they are aligned to the shock normal and, thus, obliquely propagating along the undisturbed upstream magnetic field (Mann \& Lühr 1992) with the simultaneous increasing of their amplitudes. This scenario is confirmed by the numerical particle simulations of Scholer (1993).

\section{BRIEF INTRODUCTION TO SHOCK DRIFT ACCELERATION}

In this Section we intend to derive an expression of the energy gain of a particle reflected at a shock front or a SLAMS under conservation of the magnetic moment. Here, we will follow the way given in the papers by Paschmann et al. (1980) and Schwartz et al. (1983). The frame of coordinates is chosen in such a way that the shock normal is aligned to the $x$-axis. The velocity of the reflection front is denoted by $v_{s}$. The magnetic field $B$ lies in the $x-z$ plane and takes an angle $\vartheta$ to the $x$-axis.

Now, we consider the magnetic moment conserving reflection of a particle at the shock front or the SLAMS. $V_{i}=\left(V_{i x}\right.$, $\left.V_{i y}, V_{i z}\right)=V_{i}(\cos \varphi \sin \psi, \sin \varphi \sin \psi, \cos \psi)$ denotes the incident particle velocity vector given in Cartesian and polar coordinates, respectively. After the transformation into the rest frame of the reflection front, i.e., $V_{i} \rightarrow V_{i}^{R}=\left(V_{i x}-v_{s}, V_{i y}, V_{i z}\right)$, it is useful to perform the further calculations in the de Hoffman-Teller frame. In this frame the incident velocity is given by

$$
\boldsymbol{V}_{i}^{\mathrm{HT}}=\boldsymbol{V}_{i}^{R}+\boldsymbol{v}_{\mathrm{HT}}
$$


The de Hoffman-Teller velocity $v_{\mathrm{HT}}$ is found by the conditions

$$
\begin{gathered}
\boldsymbol{V}_{i}^{\mathrm{HT}} \boldsymbol{x} \boldsymbol{B}=0 \text { and }\left(\boldsymbol{v}_{\mathrm{HT}}, \boldsymbol{x}\right)=0, \text { i.e., } \\
\boldsymbol{v}_{\mathrm{HT}}=\left(0,-V_{i y},-V_{i z}+\left\{V_{i x}-v_{s}\right\} \tan \vartheta\right) .
\end{gathered}
$$

The magnetic moment conserving reflection requires for the reflected velocity components parallel to the magnetic field $\boldsymbol{B}$ $V_{r \|}^{\mathrm{HT}}=-V_{i \|}^{\mathrm{HT}}$. Note that the magnitude of the particle velocity perpendicular to the magnetic field will be unchanged at this reflection. Now, the reflected particle velocity in the de Hoffman-Teller frame is transformed into the rest frame of the reflection front, i.e., $V_{r}^{R}=V_{r}^{\mathrm{HT}}-v_{\mathrm{HT}}$, and finally into the original frame, i.e., $V_{r}=V_{r}^{R}+v_{s} x$. In doing this, the velocity component of the reflected particle parallel to the magnetic field is found to be

$$
\begin{aligned}
V_{r \|}= & 2 v_{s} \sec \vartheta-V_{i}(\sec \vartheta) \\
& \times\left[(\cos \varphi \sin \psi)\left(1+\sin ^{2} \vartheta\right)-\cos \psi \cos \vartheta \sin \vartheta\right]
\end{aligned}
$$

In case the incident particle is moving parallel to the magnetic field, i.e., $V_{i x}=V_{i \|} \cos \vartheta$ and $V_{i z}=V_{i \|} \sin \vartheta$ as well as $\varphi=\pi$ and $\psi=\pi / 2-\vartheta$, respectively, the expression (3) reduces to equation (1) in the paper by Holman \& Pesses (1983), i.e., $V_{r \|}=2 v_{s}$ $\sec \vartheta-V_{i \|}$

The inspection of equation (3) provides that the gain of the parallel velocity of the reflected particles is mostly efficient for the particles moving toward the shock front or the SLAMS. Note that the detail reflection process is not considered here.

\section{DISCUSSION}

Assuming that SLAMS also appear at supercritical, quasiparallel shocks in the solar corona the acceleration of electrons at SLAMS is discussed under coronal circumstances, now. Here, solar Type II radio bursts are considered to be excited by supercritical shocks (cf. Benz \& Thejappa 1988). The evidence of supercritical coronal shock waves originating solar Type II radio bursts has recently been discussed by Mann et al. (1992).

Referring to the observations of solar Type II radio bursts (cf. McLean 1985) a $70 \mathrm{MHz}$ level is chosen for discussion. This plasma level corresponds to a particle number density of $6 \times 10^{7} \mathrm{~cm}^{-3}$ and is approximately located at $480,000 \mathrm{~km}$ above the photosphere according to a fourfold Newkirk density model of the solar corona (Newkirk 1961). At this level, a magnetic field of $1 \mathrm{G}$ should be expected (Dulk \& McLean 1978). This results in an ion inertial length $d_{i}=3000 \mathrm{~cm}$ and an Alfvén velocity $v_{\mathrm{A}}=280 \mathrm{~km} \mathrm{~s}^{-1}$. Adopting a coronal temperature $T=2 \times 10^{6} \mathrm{~K}$ a thermal gyroradius $r_{L}=35 \mathrm{~cm}$ is obtained with a thermal velocity $v_{\mathrm{th}}=\left(k_{B} T / m_{e}\right)^{1 / 2}=5500 \mathrm{~km} \mathrm{~s}^{-1}$ ( $k_{\mathbf{B}}$, Boltzmann's constant; $m_{e}$, electron mass) for the electrons. Since SLAMS have a spatial width of 10 ion inertial length, the condition of conserving the magnetic moment, i.e., $r_{\mathrm{L}}|\nabla \boldsymbol{B}| /|\boldsymbol{B}| \ll 1$ as well as $r_{L} \ll 10 d_{i}(\boldsymbol{B}$, magnetic field), is well fulfilled for electrons within SLAMS in the solar corona. Therefore, a conserving magnetic moment reflection of electrons at SLAMS can appropriately be expected under coronal circumstances.
Now we look to the velocity gain of electrons during their reflection at the SLAMS upstream of the shock according to equation (3). For discussing this problem, the initial velocity $\boldsymbol{V}_{i}$ should lie in a cone given by $60^{\circ} \leq \psi \leq 120^{\circ}$ and $150 \leq \varphi \leq$ $210^{\circ}$. Assuming an isotropic Maxwellian electron distribution function ahead the shock and the SLAMS, i.e.,

$$
f(v)=\left(m_{e} / 2 \pi k_{\mathrm{B}} T\right)^{3 / 2} \exp \left(-m_{e} v^{2} / k_{\mathrm{B}} T\right) ;
$$

$1 / 12$ of all electrons are put in this cone. Here, the distribution function $f(v)$ is normalized to "1." Then the number of particles $d N_{u}$ with a velocity in the interval $[v, v+d v]$ is given by

$$
d N_{u}=(2 / \pi)^{1 / 2} \mathrm{Nu}^{2} \exp \left[-u^{2} / 2\right] d u
$$

where $u$ is defined by $u=v /\left(k_{\mathrm{B}} T / m_{e}\right)^{1 / 2}$. The velocity $v_{w}$ of the most probability is chosen as the initial velocity $V_{i}$. It is found to be $u_{w}=2^{1 / 2}$ from equation (5), i.e., $v_{w}=\left(2 k_{\mathrm{B}} T / m_{e}\right)^{1 / 2}=$ $2^{1 / 2} v_{\text {th }}$, and has a value of $7800 \mathrm{~km} \mathrm{~s}^{-1}$ in the solar corona. We emphasize that $58.7 \%$ of all particles have a velocity of $v_{w}$ in the case of an isotropic Maxwellian distribution (cf. eq. [4]). Taking $\vartheta=60^{\circ}$ for the angle between the propagation direction of the SLAMS and its mean internal field (cf. Fig. 2) and $v_{s}=400$ $\mathrm{km} \mathrm{s}^{-1}$ for its speed, we find for the parallel velocity of the reflected electrons as being in the interval

$$
\begin{aligned}
18,700 \mathrm{~km} \mathrm{~s}^{-1} & =3.4 v_{\mathrm{th}} \leq V_{r \|} \leq 28,900 \mathrm{~km} \mathrm{~s}^{-1} \\
& =5.25 v_{\mathrm{th}}=0.096 c
\end{aligned}
$$

( $c$ is the velocity of light), according to equation (3). Thus, a mean value of $24,000 \mathrm{~km} \mathrm{~s}^{-1}=4.36 v_{\mathrm{th}}=0.08 \mathrm{c}$ should be expected for the parallel velocity of the reflected electrons. Only $5 \%$ of the incident particles with the velocity $v_{w}$, i.e., $0.24 \%$ of all particles, are assumed to be reflected back toward the upstream region by a SLAMS. They are accelerated to a mean velocity of $4.36 v_{\mathrm{th}}$. From the equation (4) the number of particles $d N_{u \|}$ with a velocity $v_{\|}=u_{\|}\left(k_{\mathrm{B}} T / m_{e}\right)^{1 / 2}$ parallel to the magnetic field in the interval $\left[u_{\|}, u_{\|}+d u_{\|}\right]$is derived as being

$$
d N_{u \|}=N(2 \pi)^{-1 / 2} \exp \left[-u_{\|}^{2} / 2\right] d u_{\|}
$$

Thus, $2.97 \times 10^{-5} \mathrm{~N}$ particles of the thermal background plasma have a parallel velocity of $4.36 v_{\mathrm{th}}$. Therefore, a bumpon-tail distribution can be developed by the aforementioned acceleration process well ahead of a SLAMS, i.e., in the upstream region of a quasi-parallel shock. Such a particle population is unstable and able to excite Langmuir waves (cf. Krall \& Trivelpiece 1973). Furthermore, at the maximum enhancement of the magnetic field within the SLAMS the angle $\vartheta$ is increasing to $75^{\circ}$. Then the equation (3) provides a maximum of $V_{r \|}=57,300 \mathrm{~km} \mathrm{~s}^{-1}=10.4 v_{\text {th }}=0.2 c$.

Thus SLAMS are able to accelerate thermal electrons to suprathermal velocities. These suprathermal electrons can generate radio radiation via an enhanced Langmuir turbulence (cf. the discussion in $\S 1$ ), where the electrons of the highest velocities will be responsible for the herringbones.

Benz \& Thejappa (1988) have suggested an emission mechanism of the backbone where upper hybrid waves coalesce with low-frequency ion waves into escaping electromagnetic waves. 
The upper hybrid mode is excited by a shifted ring distribution for the electrons. Such anisotropic velocity distributions of electrons result from the fast Fermi acceleration process at the quasi-perpendicular shock region (Leroy \& Mangeney 1984; Wu 1984; Benz \& Thejappa 1988). Since the magnetic field geometry is locally swung into the quasi-perpendicular regime within SLAMS, such a ring distribution of electrons may also be established in the vicinity of SLAMS, i.e., upstream of a quasi-parallel shock region. Thus, these electrons can mediately generate the backbone radiation via the mechanism proposed by Benz \& Thejappa (1988). The low-frequency ion waves also necessary for this mechanism are typical upstream phenomena at the quasi-parallel shock (cf. Paschmann et al. 1979). Since SLAMS are plane upstream structures accompanied by a density enhancement, they would radiate in a frequency band above the electron plasma frequency upstream of the shock. The width of this band is determined by the local enhancement of the electron plasma frequency within the SLAMS. SLAMS are growing from the usual upstream ULF waves and float to the shock front. Thus, a few SLAMS always appear in the upstream region of a quasi-parallel shock and can occur as different radio sources according to the aforementioned discussion. Since SLAMS have a great spatial extension parallel to the shock front (cf. the two-dimensional numerical particle simulation results of Scholer et al. 1992), SLAMS could cause the multiple lane structure often observed in solar Type II radio bursts.

A quasi-parallel shock transition should be regarded as a patchwork of SLAMS (Schwartz \& Burgess 1991). This is also confirmed by the two-dimensional numerical particle simulations of Scholer, Fujimoto, \& Kucharek (1992). On the other hand, a quasi-perpendicular shock exhibits a clearly localized shock transition. Therefore, a solar Type II burst with homogeneous emission features with regard to the backbone and the herringbones might be generated by a quasi-perpendicular coronal shock. Contrary to this, a solar Type II burst produced by a quasi-parallel shock in the corona should exhibit more irregular (or patchy) emission features.

To summarize the presented results, quasi-parallel supercritical fast magnetosonic shocks in the solar corona are able to originate Type II radio bursts by accelerating electrons to suprathermal velocities at SLAMS appearing upstream of these shocks.

The authors are indebted to G. Paschmann, who is the Principal Investigator of the AMPTE/IRM Plasma Instrument, and $W$. Baumjohann for providing the data of the 3D plasma instrument.
Axford, I. 1981, IAU Symp. 94, Origin of Cosmic Rays, ed. G. Setti, G. Spada, \& A. W. Wolfendale (Dordrecht: Reidel), 339

Benz, A. O., \& Thejappa, G. 1988, A\&A, 202, 267

Dulk, G. A., \& McLean, D. J. 1978, Sol. Phys., 57, 279

Fermi, E. 1949, Phys. Rev., 75, 1149

Gurnett, D. A. 1985, in Plasma Waves and Instabilities in Collisionless Shocks in the Heliosphere; Reviews of Current Research, ed. B. T. Tsurutani \& R. G. Stone (Washington: AGU, GN-34), 207

Holman, G. D., \& Pesses, M. E. 1983, ApJ, 267, 837

Kennel, C. F., Edmiston, J. P., \& Hada, T. 1985, A Quarter Century in Collisionless Shocks in Collisionless Shocks in the Heliosphere: Reviews of Current Research, ed. B. T. Tsurutani \& R. G. Stone (Washington: AGU, GN-34), 1

Kennel, C. F., Scarf, F. L., Coroniti, F. V., Smith, E. J., \& Gurnett, D. A. 1982, J. Geophys. Res., 87, 17

Krall, N. A., \& Trivelpiece, A. W. 1973, Principles of Plasma Physics (New York: McGraw Hill)

Krüger, A. 1979, Introduction to Solar Radio Astronomy and Radio Physics (Dordrecht: Reidel)

Leroy, M. M., \& Mangeney, A. 1984, Ann. Geophys., 2, 449

Lühr, H., Klöcker, N., Oelschlägel, W., Häusler, B., \& Acuna, M. 1985, IEEE Trans. Geosci. Remote Sens., GE-23, 259

Mann, G., Aurass, H., Voigt, W., \& Paschke, J. 1992, ESA Journal, ESA SP-348, 129

Mann, G., \& Lühr, H. 1992, ESA Journal, ESA SP-346, 91

McLean, D. J. 1985, in Solar Radiophysics, ed. D. J. McLean \& N. R. Labrum (Cambridge Univ. Press), 37
Newkirk, G. A. 1961, ApJ, 133, 983

Parks, G. K., et al. 1981, J. Geophys. Res., 86, 4343

Paschmann, G., Loidl, H., Obermayer, F., Erbl, M., Laborenz, R., Sckopke, N., Baumjohann, W., \& Carlson, C. W. 1985, IEEE Trans. Geosci. Remote Sens., GE-23, 262

Paschmann, G., Sckopke, N., Asbridge, J. R., Bame, S. J., \& Gosling, J. T. 1980, J. Geophys. Res., 85, 4689

Paschmann, G., Sckopke, N., Bame, S. J., Asbridge, J. R., Gosling, J. T., Russell, C. T., \& Greenstadt, E. W. 1979, Geophys. Res. Lett., 6, 209

Scholer, M. 1985, Diffuse Shock Acceleration in Collisionless Shocks in the Heliosphere: Reviews of Current Research, ed. B. T. Tsurutani \& R. G. Stone (Washington: AGU, GN-34), 287

- 1993, J. Geophys. Res., 98, 47

Scholer, M., Fujimoto, M., \& Kucharek, H. 1992, ESA Journal, ESA SP346,59

Schwartz, S. J., \& Burgess, D. 1991, Geophys. Res. Lett., 18, 373

Schwartz, S. J., Burgess, D., Wilkinson, W. P., Kessel, R. L., Dunlop, M., \& Lühr, H. 1992, J. Geophys. Res., 97, 4209

Schwartz, S. J., Thomsen, M. F., \& Gosling, J. T. 1983, J. Geophys. Res., 88,2039

Thomson, M. F., Gosling, J. T., Bame, S. J., \& Russel, C. T. 1990, J. Geophys Res., 95, 957

Tsurutani, B. T., \& Rodriguez, P. 1981, J. Geophys. Res., 86, 4319

Wu, C. S. 1984, J. Geophys. Res., 89, 8857 